References: 1. Munira S, Christopher-Stine L. Pregnancy in myositis and scleroderma. Best Pract Res Clin Obstet Gynaecol. 2020 Apr;64:59-67. doi: 10.1016/j. bpobgyn.2019.10.004. Epub 2019 Oct 18. PMID: 31928915.

Disclosure of Interests: None declared

DOI: 10.1136/annrheumdis-2021-eular.1539

\section{POS1425 ANTIBODIES TO PORPHYROMONAS GINGIVALIS ASSOCIATE WITH THE PRESENCE OF RHEUMATOID ARTHRITIS-RELATED AUTOANTIBODIES IN PATIENTS WITH PERIODONTITIS}

C. De Vries ${ }^{1}$, G. Ruacho ${ }^{1}$, N. Sippl', B. A. Potempa ${ }^{2}$, L. Ryden ${ }^{3}$, J. S. Potempa ${ }^{2}$, B. Klinge ${ }^{4}$, E. Svenungsson ${ }^{1}$, K. Lundberg ${ }^{1}{ }^{1}$ Karolinska Institute, Department of Medicine, Division of Rheumatology, Stockholm, Sweden; ${ }^{2}$ University of Louisville, Department of Oral Immunology \& Infectious Diseases, Louisville, United States of America; ${ }^{3} \mathrm{Nya}$ Karolinska, Department of Medicine, Stockholm, Sweden; ${ }^{4}$ Huddinge Hospital, Department of Dental Medicine, Division of Oral Diseases, Huddinge, Sweden

Background: Epidemiologic studies have demonstrated a link between periodontitis (PD) and rheumatoid arthritis (RA), specifically RA characterized by anti-citrullinated protein antibodies (ACPA). The keystone pathogen driving PD, Porphyromonas gingivalis $(P g)$, is the only pathogen known to express peptidylarginine deiminase (PAD), a citrullinating enzyme. Hence, $P g$ has been proposed to be involved in triggering the ACPA response, by generating citrullinated antigens in an inflammatory milieu(1). Another major virulence factor of $P g$ is arginine gingipain B (RgpB), a proteinase which cleaves proteins so that $P$.PAD can access the site where citrullination takes place. We have previously shown elevated anti-RgpB IgG levels in ACPA+ RA patients, even before clinical onset(2,3), and we hypothesize that anti-RgpB IgG could serve as a serological marker to identify PD patients with increased risk of developing ACPA+ RA.

Objectives: Based on this hypothesis, we set out to investigate whether antiRgpB IgG was associated with PD, PD severity, autoimmunity in general, and the ACPA response in particular.

Methods: Anti-RgpB IgG, as well as RA- and systemic lupus erythematosus (SLE)-related autoantibodies targeting cyclic citrullinated peptide(s) (CCP2), rheumatoid factor (RF), dsDNA, cardiolipin, and $\beta 2$ glycoprotein, were measured by ELISA in serum samples from the ParoKrank study, which is a well-characterized cohort of 805 patients with a first myocardial infarction and 805 matched controls, where periodontal status has been determined by dentists(4). In this study, individuals with PD $(n=941)$ were compared to individuals without PD $(n=557)$.

Results: We detected significantly elevated $(p<0,0001)$ anti-RgpB IgG levels in $P D$ compared to non-PD individuals, with highest levels recorded in severe PD. Anti-RgpB IgG levels were significantly increased in PD patients positive for CCP2 and/or RF ( $n=50)$, when compared to PD patients negative for CCP2 and RF ( $n=507), p<0,05$, and when compared to non-PD individuals positive for CCP2 and/or RF ( $n=62), p<0,05$. Notably, these differences were not seen for SLE-related autoantibodies. In addition, anti-RgpB IgG levels were significantly elevated amongst MI patients versus controls $(p<0,05)$, supporting the previous finding that $\mathrm{PD}$ is more common among MI patients(4).

Conclusion: Our data demonstrates a specific association between severe PD, elevated anti-RgpB IgG levels and RA-related autoantibodies, supporting a role for $P g$ in linking PD to ACPA+ RA. Further investigation will be needed to confirm whether anti-RgpB IgG can be used as a serological marker to identify PD patients with increased risk of developing ACPA+ RA.

\section{REFERENCES:}

[1] Rosenstein ED, Greenwald RA, Kushner LJ, Weissmann G. Hypothesis: the humoral immune response to oral bacteria provides a stimulus for the development of rheumatoid arthritis. Inflammation. 2004;28(6):311-8.

[2] Kharlamova N, Jiang X, Sherina N, Potempa B, Israelsson L, Quirke AM, et al. Antibodies to Porphyromonas gingivalis Indicate Interaction Between Oral Infection, Smoking, and Risk Genes in Rheumatoid Arthritis Etiology. Arthritis Rheumatol. 2016;68(3):604-13.

[3] Johansson L, Sherina N, Kharlamova N, Potempa B, Larsson B, Israelsson $\mathrm{L}$, et al. Concentration of antibodies against Porphyromonas gingivalis is increased before the onset of symptoms of rheumatoid arthritis. Arthritis Res Ther. 2016;18(1):201.

[4] Rydén L, Buhlin K, Ekstrand E, Faire Ud, Gustafsson A, Holmer J, et al. Periodontitis Increases the Risk of a First Myocardial Infarction. Circulation. 2016;133(6):576-83.

Disclosure of Interests: None declared

DOI: 10.1136/annrheumdis-2021-eular.1580

\section{POS1426 PATIENTS WITH IMMUNE MEDIATED INFLAMMATORY DISEASES ARE OVERREPRESENTED IN LOW- FREQUENCY VIRAL SYMPTOM CLUSTERS}

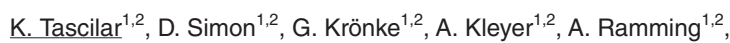
R. Atreya ${ }^{2,3}$, M. Tenbusch ${ }^{4}$, K. Überla ${ }^{4}$, C. Berking ${ }^{2,5}$, M. Sticherling ${ }^{2,5}$, M. F. Neurath ${ }^{2,3}$, G. Schett ${ }^{1,2} .{ }^{1}$ Friedrich Alexander University of Erlangen Nuremberg and Universitätsklinikum Erlangen, Department of Medicine-3, Rheumatology and Immunology, Erlangen, Germany; ${ }^{2}$ Friedrich Alexander University of Erlangen Nuremberg and Universitätsklinikum Erlangen, Deutsches Zentrum für Immuntherapie, Erlangen, Germany; ${ }^{3}$ Friedrich Alexander University of Erlangen Nuremberg and Universitätsklinikum Erlangen, Department of Medicine-1, Gastroenterology, Pneumology and Endorcrinology, Erlangen, Germany; ${ }^{4}$ Friedrich Alexander University of Erlangen Nuremberg and Universitätsklinikum Erlangen, Institute of Clinical and Molecular Virology, Erlangen, Germany; ${ }^{5}$ Friedrich Alexander University of Erlangen Nuremberg and Universitätsklinikum Erlangen, Department of Dermatology, Erlangen, Germany

Background: Viral respiratory infections are common in the general population and result in a spectrum of outcomes ranging from effective viral clearance with no symptoms, to a maladaptive immune response that can result in severe symptomatic disease and death. Although patients with immune-mediated inflammatory diseases (IMID) are considered susceptible to poor outcomes from infectious syndromes, it is not known whether IMID patients are overall more prone to manifest common viral infection symptoms.

Objectives: To explore frequency patterns of common viral infection symptoms in IMID patients.

Methods: We previously recruited patients with IMIDs and individuals with no IMIDs for a seroprevalence study between February $1^{\text {st }}$ and April $30^{\text {th }} 2020$ (1). Participants were questioned for the presence of eleven common viral disease symptoms. We clustered these data using an unsupervised binary data clustering algorithm (2) into 6 symptom clusters based on symptom frequency. Three major clusters (broadly symptomatic, intermediately symptomatic and oligo-/ asymptomatic) and 2 sub-clusters (higher and lower frequency) for each major cluster. In addition, qualitative symptom clustering was done. We estimated standardized residuals to quantify the over/underrepresentation of IMID diagnosis frequencies in each subject cluster. We used Poisson regression to compare symptom counts by diagnosis.

Results: We analyzed 1909 participants (757 with IMIDs; 1152 non-IMID controls; Table 1). Within each major subject cluster (Figure 1A), IMID patients showed the highest positive deviation from the expected frequencies in lower frequency sub-clusters while non IMID controls showed the highest positive deviations in the higher frequency sub-clusters (Figure 1B). Inflammatory bowel disease and psoriasis were remarkably overrepresented in the lower frequency sub-cluster of the broadly-symptomatic cluster while RA was overrepresented in the lower frequency sub-clusters of intermediate and oligo-/asymptomatic clusters. $X$ axis of Figure $1 \mathrm{~A}$ presents qualitative symptom clusters. Regression analysis shows that RA patients among other IMIDs reported overall less symptoms ( $R R=0.69,95 \% \mathrm{Cl}, 0.58-0.80$ ) compared to non-IMID controls.

Table 1. Participant characteristics and distribution of IMID diagnoses across subject clusters.

\begin{tabular}{|c|c|c|c|c|c|c|c|}
\hline & & \multicolumn{6}{|c|}{ Clusters } \\
\hline & & \multicolumn{2}{|c|}{$\begin{array}{c}\text { Broad } \\
\text { Symptomatic }\end{array}$} & \multicolumn{2}{|c|}{$\begin{array}{l}\text { Intermediate } \\
\text { Symptomatic }\end{array}$} & \multicolumn{2}{|c|}{$\begin{array}{c}\text { Oligo- } \\
\text { Asymptomatic }\end{array}$} \\
\hline & Overall & Higher & Lower & Higher & Lower & Higher & Lower \\
\hline $\mathbf{N}$ & 1909 & 101 & 85 & 412 & 259 & 283 & 769 \\
\hline $\begin{array}{l}\text { Age, years, } \\
\text { mean (SD) }\end{array}$ & $\begin{array}{c}45.4 \\
(15.2)\end{array}$ & $\begin{array}{c}42.4 \\
(13.3)\end{array}$ & $\begin{array}{c}47.3 \\
(15.2)\end{array}$ & $\begin{array}{c}42.4 \\
(12.9)\end{array}$ & $\begin{array}{c}50.4 \\
(15.5)\end{array}$ & $\begin{array}{c}41.8 \\
(14.9)\end{array}$ & $\begin{array}{c}46.8 \\
(15.9)\end{array}$ \\
\hline Male & $\begin{array}{l}1080 \\
(56.6)\end{array}$ & $\begin{array}{c}42 \\
(41.6)\end{array}$ & $\begin{array}{c}38 \\
(44.7)\end{array}$ & $\begin{array}{c}196 \\
(47.6)\end{array}$ & $\begin{array}{c}137 \\
(52.9)\end{array}$ & $\begin{array}{c}178 \\
(62.9)\end{array}$ & $\begin{array}{c}489 \\
(63.6)\end{array}$ \\
\hline Diagnosis, n(\%) & & & & & & & \\
\hline No-IMID & $\begin{array}{c}1152 \\
(60.3)\end{array}$ & $\begin{array}{c}72 \\
(71.3)\end{array}$ & $\begin{array}{c}44 \\
(51.8)\end{array}$ & $\begin{array}{c}280 \\
(68.0)\end{array}$ & $\begin{array}{c}112 \\
(43.2)\end{array}$ & $\begin{array}{c}207 \\
(73.1)\end{array}$ & $\begin{array}{c}437 \\
(56.8)\end{array}$ \\
\hline RA & $226(11.8)$ & $7(6.9)$ & $5(5.9)$ & $29(7.0)$ & $56(21.6)$ & $17(6.0)$ & $112(14.6)$ \\
\hline IBD & 178 (9.3) & $5(5.0)$ & 15 (17.6) & 46 (11.2) & 29 (11.2) & $19(6.7)$ & $64(8.3)$ \\
\hline SpA & $142(7.4)$ & $7(6.9)$ & $5(5.9)$ & $23(5.6)$ & $25(9.7)$ & $14(4.9)$ & $68(8.8)$ \\
\hline Psoriasis & $89(4.7)$ & $4(4.0)$ & $9(10.6)$ & $14(3.4)$ & $8(3.1)$ & $13(4.6)$ & $41(5.3)$ \\
\hline Other & $122(6.4)$ & $6(5.9)$ & $7(8.2)$ & $20(4.9)$ & $29(11.2)$ & $13(4.6)$ & $47(6.1)$ \\
\hline $\begin{array}{l}\text { Symptom count/ } \\
\text { patient, mean (SD) }\end{array}$ & $1.2(1.7)$ & $6.0(1.3)$ & $3.9(1.1)$ & $2.2(1.0)$ & $1.5(0.6)$ & $0.5(0.5)$ & $0.0(0.0)$ \\
\hline
\end{tabular}




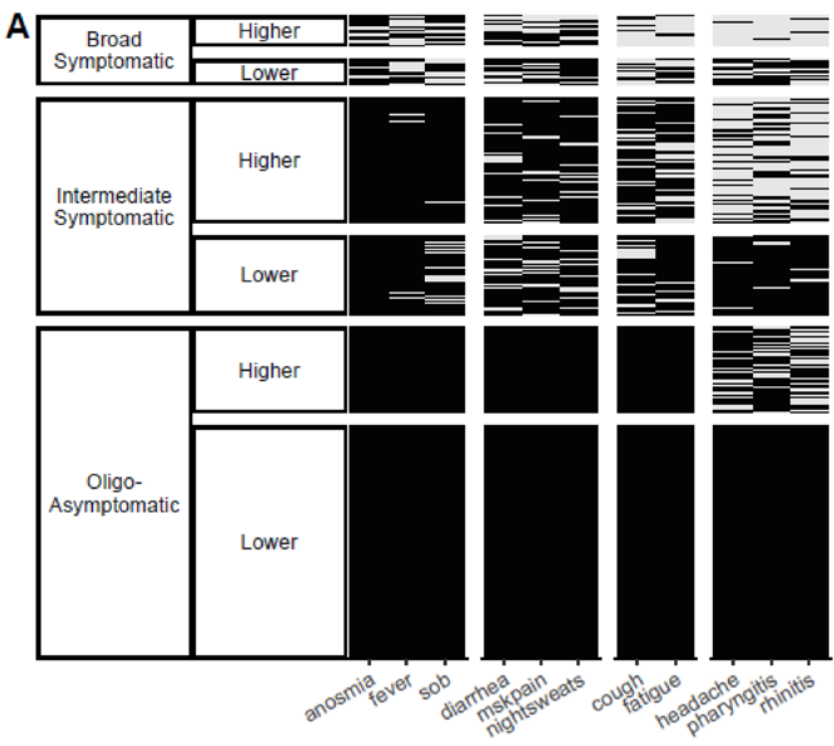

B

Broad Symptomatic
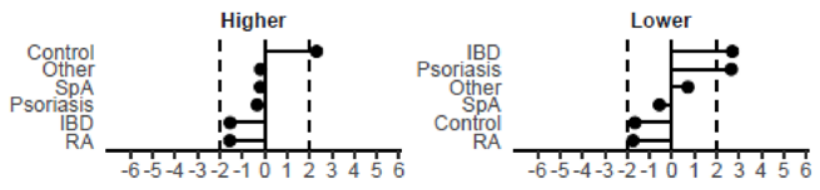

Intermediate Symptomatic
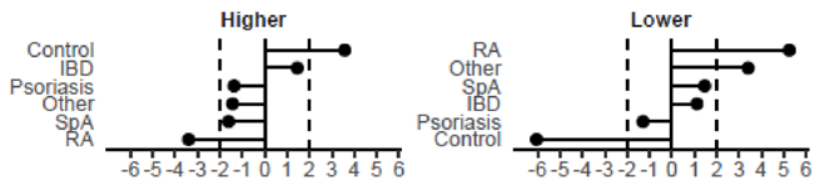

Oligo-/Asymptomatic

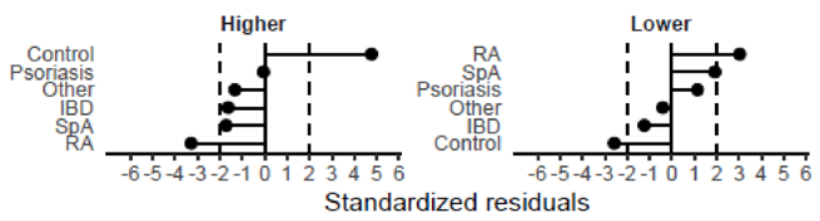

Figure 1. A) distribution of common viral respiratory disease symptoms across patient and symptom clusters. B) Standardized residuals indicating deviation from expected frequencies of IMID diagnoses across patient clusters. sob: shortness of breath, mskpain: musculoskeletal pain

Conclusion: This analysis shows that symptoms of common respiratory viral infections are less frequent in RA patients and to a lesser extent in other IMID patient. As major clusters in this analysis can also be considered to represent exposure categories, these data suggest that IMIDs or their treatments may mitigate common respiratory viral infection symptoms.

REFERENCES:

[1] Simon D. et al. Nat Commun (2020) 11, 3774

[2] Bhatia P. et al. J. Stat. Softw (2017) 76(9)

Acknowledgements: This study was supported by the Deutsche Forschungsgemeinschaft (DFG- FOR2886 PANDORA and the CRC1181), the Bundesministerium für Bildung und Forschung (BMBF; project MASCARA), the H2020 GA 810316 - 4D-Nanoscope ERC Synergy Project, the IMI funded project RTCure, the Emerging Fields Initiative MIRACLE of the Friedrich-AlexanderUniversität Erlangen-Nürnberg as well as the Schreiber Stiftung gemeinnützige Gesellschaft $\mathrm{mbH}$.

Disclosure of Interests: None declared

DOI: 10.1136/annrheumdis-2021-eular.1598

\section{POS1427 NATIONAL BURDEN OF RHEUMATOID ARTHRITIS IN CANADA 1990-2017: FINDINGS FROM THE GLOBAL BURDEN OF DISEASE STUDY 2017}

N. Hassen ${ }^{1}$, D. Lacaille ${ }^{1}$, A. Xu ${ }^{1}$, S. Sidi ${ }^{1}$, A. Alandejani ${ }^{1}$, M. Mansourian ${ }^{2}$, N. Sarrazadegan ${ }^{1}$, J. Kopec ${ }^{1} .{ }^{1}$ The University of British Columbia, Faculty of Medicine, Vancouver, Canada; ${ }^{2}$ Isfahan University of Medical Sciences, Isfahan, Iran (Islamic Republic of)

Background: According to the Global Burden of Diseases, Injuries, and Risk Factors Study (GBD) 2017, over 120,000 individuals currently have rheumatoid arthritis (RA) in Canada, yet a study that evaluates the combined effect of RA on the longevity and quality of life in the country is lacking.

Objectives: The objectives of this study are three: 1) to describe burden of RA levels and trends from 1990-2017 using GBD data, 2) to describe age and sex differences, and 3) to compare Canada RA burden to other countries.

Methods: We obtained publicly available data from GBD Study 2017 from the Institute for Health Metrics and Evaluation interactive visualization tool (http:// vizhub.healthdata.org/gbd-compare). Disease burden indicators include prevalence, mortality, years of life lost (YLLs), years lived with disability (YLDs), and disability-adjusted life years (DALYs). Estimates were presented as non age-standardized and age-standardized rates per 100,000 population. GBD estimated prevalence using published literature, survey data, patient records, and health insurance claims, and mortality using cause of death ensemble modelling technique. YLLs measure premature death calculated as the sum of each death multiplied by the standard life expectancy at each age. YLDs measure amount of time in a year an individual lives with a short- or long-term health condition, calculated by combining prevalence with disability weights for each age/sex/year. DALYs were calculated as the sum of YLLs and YLDs. DALYs for Canada were compared to DALYs of countries with similar socio-demographic index (SDI) values. SDI combines income per capita, average educational attainment, and fertility rates. Data were analysed by DisMod-MR 2.1, a Bayesian meta-regression tool.

Results: In Canada, RA mortality (mortality and YLLs) improved over time, with a steeper decline after the year 2002. However, the population burden of quality of life (YLDs and DALYs) increased due to increasing prevalence. The disease burden was higher in females (prevalence, mortality, YLLs, YLDs, DALYs), and DALY rates were higher among older populations. Compared to other countries, Canada had greater improvement in mortality and YLLs over time and had a lower age-standardized DALYs rate compared to countries of similar SDI values. A weak association was found between global age-standardized DALYs and SDI $\left(R^{2}=0.0138\right)$

Conclusion: RA is a major public health challenge. Canada fares better than other countries with regards to national RA burden. Early identification and management are critical to reducing the overall burden of RA in Canada, especially in women. More data from multiple provincial RA databases would increase the accuracy of our estimates for Canada.

\section{REFERENCES:}

[1] Cross M, Smith E, Hoy D, et al. The global burden of rheumatoid arthritis: estimates from the global burden of disease 2010 study. Ann Rheum Dis. 2014;73(7):1316-1322. doi:10.1136/annrheumdis-2013-204627

[2] Myasoedova E, Davis JM 3rd, Crowson CS, Gabriel SE. Epidemiology of rheumatoid arthritis: rheumatoid arthritis and mortality. Curr Rheumatol Rep. 2010;12(5):379-385. doi:10.1007/s11926-010-0117-y

[3] Safiri S, Kolahi AA, Hoy D, et al. Global, regional and national burden of rheumatoid arthritis 1990-2017: a systematic analysis of the Global Burden of Disease study 2017. Ann Rheum Dis. 2019;78(11):1463-1471. doi:10.1136/ annrheumdis-2019-215920

Acknowledgements: Nejat Hassen is supported by a grant from the Canadian Institute of Health Research. Dr. Diane Lacaille is supported by the Mary Pack Chair in Arthritis Research from the University of British Columbia and The Arthritis Society of Canada.

Disclosure of Interests: None declared

DOI: 10.1136/annrheumdis-2021-eular.1927

\section{POS1428 VALIDATION OF ANCA-ASSOCIATED VASCULITIS AS THE CAUSE OF END-STAGE RENAL DISEASE IN THE UNITED STATES RENAL DATA SYSTEM}

C. Cook ${ }^{1}$, H. Choi ${ }^{1,2}$, Z. Wallace ${ }^{1,2} .{ }^{1}$ Massachusetts General Hospital, Division of Rheumatology, Allergy, and Immunology, Boston, United States of America;

${ }^{2}$ Harvard, Medical School, Boston, United States of America

Background: Glomerulonephritis and other renal manifestations are common in ANCA-associated vasculitis (AAV). Renal involvement in AAV is associated with adverse outcomes, including end-stage renal disease (ESRD) in up to $25 \%$ of patients (1). The United States Renal Data System (USRDS), a national registry of ESRD patients, represents a unique nationwide data source for studying AAV patients with ESRD. Prior research has assessed how often patients with ESRD attributed to AAV have biopsy-proven glomerulonephritis in USRDS (2), but the validity of the diagnosis of AAV as the cause of ESRD in the USRDS remains unknown.

Objectives: We aim to validate the diagnosis of AAV as the primary cause of ESRD listed in USRDS. 